\title{
Glycan-binding F-box protein from Arabidopsis thaliana protects plants from Pseudomonas syringae infection
}

\author{
Karolina Stefanowicz ${ }^{\dagger}$, Nausicaä Lannoo ${ }^{\dagger}$, Yafei Zhao, Lore Eggermont, Jonas Van Hove, Bassam Al Atalah
} and Els J. M. Van Damme*

\begin{abstract}
Background: A small group of F-box proteins consisting of a conserved F-box domain linked to a domain homologous to the glycan-binding protein has been identified within the genome of Arabidopsis thaliana. Previously, the so-called F-box-Nictaba protein, encoded by the gene At2902360, was shown to be a functional lectin which binds $N$-acetyllactosamine structures. Here, we present a detailed qRT-PCR expression analysis of F-box-Nictaba in Arabidopsis plants upon different stresses and hormone treatments.

Results: Expression of the F-box-Nictaba gene was enhanced after plant treatment with salicylic acid and after plant infection with the virulent Pseudomonas syringae pv. tomato strain DC3000 (Pst DC3000). $\beta$-glucuronidase histochemical staining of transgenic Arabidopsis plants displayed preferential activity of the At2g02360 promoter in trichomes present on young rosette leaves. qRT-PCR analyses confirmed high expression of F-box-Nictaba in leaf trichomes. A. thaliana plants overexpressing the gene showed less disease symptoms after Pst DC3000 infection with reduced bacterial colonization compared to infected wild type and F-box-Nictaba knock-out plants.

Conclusions: Our data show that the Arabidopsis F-box-Nictaba gene is a stress-inducible gene responsive to SA, bacterial infection and heat stress, and is involved in salicylic acid related plant defense responses. This knowledge enriched our understanding of the physiological importance of F-box-Nictaba, and can be used to create plants with better performance in changing environmental conditions.
\end{abstract}

Keywords: F-box-Nictaba, Glycan, Lectin, Pseudomonas, Salicylic acid, Stress

\section{Background}

F-box proteins represent one of the largest and most diverse protein families in plants, as most species have several hundred representatives [1]. They are named after their highly conserved $\mathrm{N}$-terminal protein-protein interaction motif of approximately 50 amino acid residues, known as the F-box domain. The majority of the F-box proteins function as part of Skp1-Cullin1-F-box (SCF)-type ubiquitin E3 ligases [2] in which the F-box protein comprises the substrate-binding module. F-box proteins are assembled into active SCF complexes through a direct binding of the F-box motif with the SCF core S-phase kinase-related

\footnotetext{
* Correspondence: elsjm.vandamme@ugent.be

${ }^{\dagger}$ Equal contributors

Department of Molecular Biotechnology, Ghent University, Coupure Links 653, B-9000 Ghent, Belgium
}

protein 1 (Skp1). Through their variable C-terminal substrate-binding domain, F-box proteins specifically bind to and deliver appropriate substrates to the SCF complex for ubiquitin-mediated proteolysis by the ubiquitinproteasome system [3].

The impressive number of F-box proteins in plants and extensive diversity of their C-terminal target-binding omains contribute to the ability of SCF complexes to arget a wide variety of substrates. Therefore, it is not prising that F-box proteins are involved in numerous ular processes within plant development and stress signg [4]. Genetic approaches already revealed an essential or F-box proteins in plant hormone perception and signaling [5]. F-box proteins were also reported to be involved in circadian clock control, photomorphogenesis 
and flowering [6, 7], leaf senescence [8], self-incompatibility [9] and responses to various (a)biotic stresses [10-12].

Multiple newly discovered F-box proteins (the so-called FBXO proteins) combine various recognition mechanisms which enable tight regulation of substrate selection by the F-box protein and each complementary SCF complex [3]. Amongst these new FBXO proteins, glycan-binding F-box (or FBG) proteins were first discovered in mammals [13], including FBG1, FBG2 and FBG5 which bind to highmannose $N$-glycosylated proteins [14]. The C-terminal substrate-binding domain of both FBG1 and FBG2 specifically interacts with the inner $\mathrm{N}, \mathrm{N}^{\prime}$-diacetylchitobiose $\left(\mathrm{GlcNAc}_{2}\right)$ core of high-mannose $\mathrm{N}$-glycans present on incompletely folded or denatured glycoproteins. Since FBG1 and FBG2 do not target free mannose structures or non-glycosylated proteins, it was concluded that both FBG proteins function as glycan-binding F-box proteins involved in the endoplasmic reticulum associated degradation pathway [15]. In this pathway, proteins which fail to fold correctly or assemble into oligomeric complexes in the lumen of the endoplasmic reticulum are retro-translocated to the cytosol, where they are captured by an $\mathrm{SCF}^{\mathrm{FBG1} / 2}$ complex before degradation by the ubiquitin-proteasome system [16].

Recently, FBG-like proteins were also reported in plants. The $A$. thaliana genome contains multiple genes encoding F-box proteins with a putative glycan-binding or lectinlike domain $[17,18]$. These proteins are referred to as the F-box-Nictaba family, since their C-terminal domain highly resembles Nictaba, an $\mathrm{N}$-glycan-binding jasmonateinducible lectin from tobacco [19]. This F-box protein family in A. thaliana groups approximately 30 members that share over 90 and $40-64 \%$ sequence similarity in the F-box domain and the Nictaba domain, respectively [4]. Due to the presence of the F-box domain linked to a lectin-like C-terminal substrate-binding domain, it is tempting to speculate that these F-box-Nictaba proteins function as substrate adaptors in an endoplasmic reticulum associated degradation pathway in plants similar to the mammalian FBG proteins. Furthermore, both Nictaba and FBG1 show comparable glycan-binding properties towards the inner core structure of $\mathrm{N}$-glycans $[14,20]$. However, detailed analysis of the sugar specificities for the $A$. thaliana F-box-Nictaba protein (encoded by the gene At2g02360) revealed binding of its Nictaba domain to $\mathrm{N}$ - and $\mathrm{O}$-glycans containing (poly) $\mathrm{N}$-acetyllactosamine (Galß1-3GlcNAc and Galß1-4GlcNAc) structures, Lewis a, Lewis $\mathrm{x}$ and Lewis y motifs [18]. At present, it is not clear if and how the (nucleocytosolic) F-box-Nictaba protein can bind to these structures in planta. $\mathrm{N}$-glycan modification with Lewis a motifs takes place in the Golgi apparatus and plant-specific Lewis epitopes were only shown on some extracellular glycoproteins and on membrane-bound glycoproteins present at the plant cell surface [21-23]. Moreover, in contrast to most plant families, the occurrence of Lewis a motifs in A. thaliana was reported to be generally low and tissue specific with highest levels in pedicels, stems and nodes and with moderate levels in siliques and the shoot apex. Lewis a structures are not detectable in the leaves of A. thaliana plants [24].

In this study, we show that the transcripts for the $A$. thaliana F-box-Nictaba protein encoded by At2g02360 are up-regulated after plant treatment with salicylic acid (SA), heat stress and upon infection with Pseudomonas syringae pv. tomato strain DC3000, with preferential activity of its promoter sequence in leaf trichomes. Stress experiments on transgenic $A$. thaliana plants with altered F-boxNictaba expression suggested a role for F-box-Nictaba in plant defense-related signaling pathways.

\section{Results}

F-box-Nictaba gene expression is up-regulated after SA application and heat shock treatment

The expression pattern of F-box-Nictaba was first analysed in wild-type (WT) A. thaliana plants grown under laboratory growth conditions. qRT-PCR analyses demonstrated that At2g02360 was expressed during all developmental stages and in every tissue tested (Fig. 1a). A slightly lower expression of At2g02360 was observed in roots from young plants and in flowers compared to the youngest stage tested (i.e., cotyledons stage). In silico expression analysis indicated that $A t 2 g 02360$ is a stressresponsive gene (Additional file 1: Figures S1-S2, Table S1 and Text S1). Indeed, the uniform expression profile of At2g02360 considerably changed when $A$. thaliana plants were subjected to treatments with various plant hormones and several abiotic stress factors (Fig. 1b-c) (Additional file 1: Figure S4). After $1 \mathrm{~h}$ (h) of SA treatment, At2g02360 expression was slightly down-regulated compared to the mock treatment, but At2g02360 mRNA levels were significantly higher after $3 \mathrm{~h}$ with a maximal increase of almost 4-fold after $10 \mathrm{~h}$ of SA application (Fig. 1b). Transcript levels in treated plants slightly dropped after $24 \mathrm{~h}$ but were still two-fold higher than in the untreated plants. Transcript levels for the SA-inducible transcription factor WRKY70 were up-regulated faster and reached much higher levels compared to F-box-Nictaba expression levels (Additional file 1: Figure S3a).

Also heat stress enhanced F-box-Nictaba gene expression with a maximal up-regulation of almost 3-fold after $10 \mathrm{~h}$ of heat stress compared to control plants (Fig. 1c). However, compared to the At2g02360 gene the expression of the $H s p 70 b$ gene, encoding a heat shock-responsive chaperone protein, was enhanced much faster and reached much higher mRNA levels (Additional file 1: Figure S3b). Other hormones and abiotic stress treatments either slightly down-regulated or did not affect F-box-Nictaba gene expression at all (Additional file 1: Figure S4). 

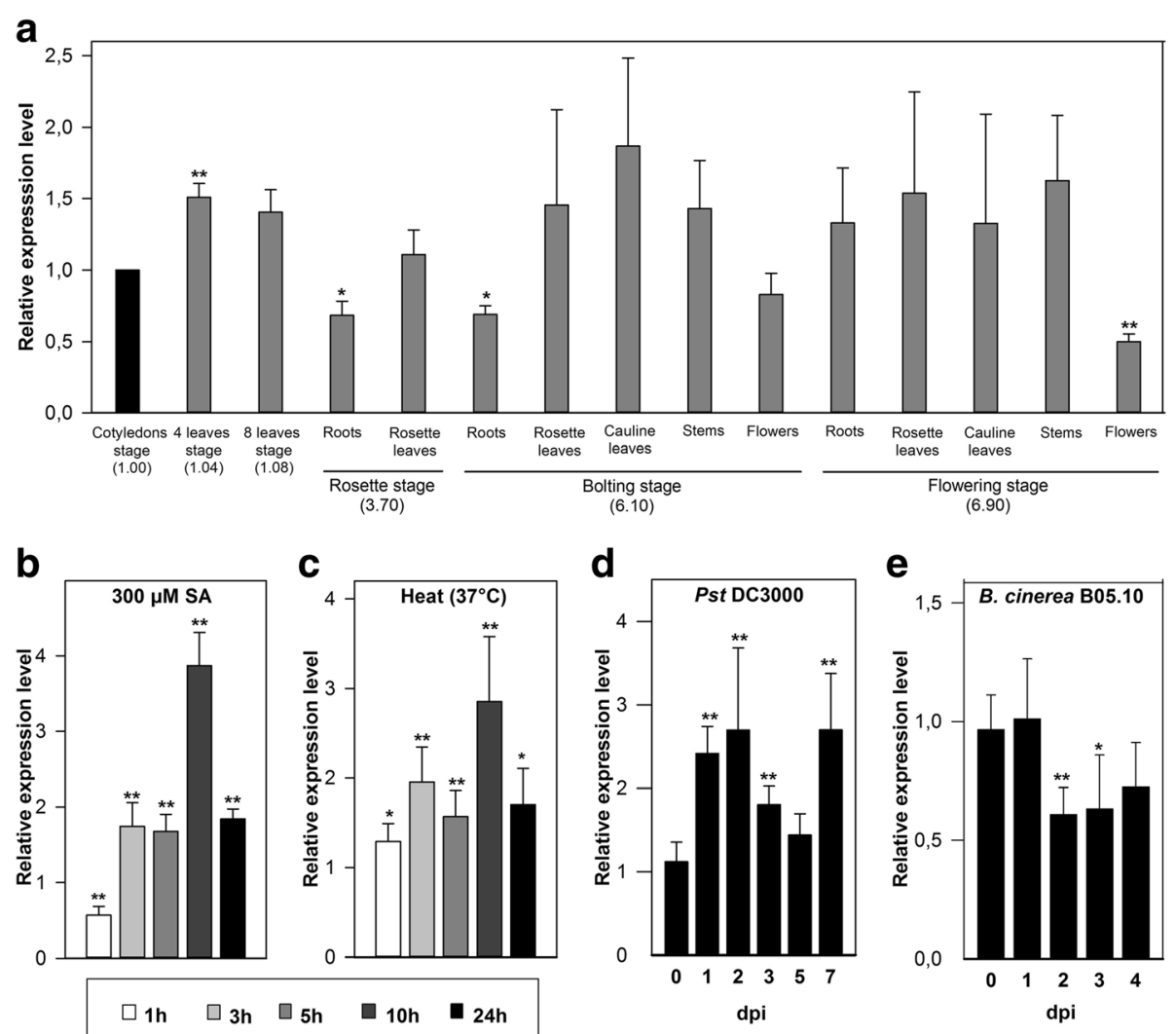

Fig. 1 Relative transcript levels of At2g02360 in WT A. thaliana Col-0 plants determined by qRT-PCR analyses of two independent biological experiments, each with multiple technical replicates as pointed out in Methods section; error bars $\pm S E$. Asterisks indicate statistically significant differential expression compared to control samples ( $\left.{ }^{*} p<0.05 ;{ }^{* *} p<0.01\right)$. a, At2g02360 expression levels measured in A. thaliana plants grown under standard conditions. b-c, At2g02360 expression levels in 16-day-old A. thaliana seedlings after SA treatment (b) or after heat shock (c). d-e, Relative expression of At2g02360 after infection of 5-week-old A. thaliana plants with Pst DC3000 (d) or with B. cinerea strain B05.10 (e). Expression levels are compared (a) to the At2g02360 expression level in the cotyledons stage or (b-e) to gene expression levels in the mock-treated plants

F-box-Nictaba expression is up-regulated in WT A. thaliana plants after Pseudomonas infection but is slightly down-regulated by fungal infection

Five-week-old WT $A$. thaliana Col-0 plants were infected either with the virulent hemibiotrophic bacterium P. syringae pv. tomato strain DC3000 (Pst DC3000) or the necrotrophic fungus Botrytis cinerea strain B05.10. Pst DC3000 infection strongly enhanced the expression of both control genes WRKY70 and PR1 in rosette leaves (Additional file 1: Figure S3c). F-boxNictaba mRNA levels were significantly up-regulated approximately 2.5 -fold at 1,2 and $7 \mathrm{dpi}$ compared to mock-sprayed plants. F-box-Nictaba expression dropped at 3 and $5 \mathrm{dpi}$ but was still higher than in the mocktreated plants (Fig. 1d). In contrast, B. cinerea infection mildly affected F-box-Nictaba mRNA levels compared to mock-treated plants (Fig. 1e). At 2 and 3 dpi, At2g02360 expression levels were reduced almost two-fold. The expression of the control gene PR1 was not altered significantly (Additional file 1: Figure S3d). In contrast, the expression of the control gene PDF1.2 was highly up-regulated reaching a 50-fold increase in infected tissues at 3 dpi (Additional file 1: Figure S3d).

\section{F-box-Nictaba promoter is particularly active in leaf trichomes}

To test the activity of the $5^{\prime}$-upstream region of the At2g02360 gene, a promoter At2g02360:GUS reporter construct was introduced into $A$. thaliana Col-0 plants and used for histochemical assays. First data revealed a distinct and localized GUS staining in the leaf trichomes of 14-day-old pAt2g02360:GUS A. thaliana seedlings (Fig. 2a). Subsequently, plants grown under standard conditions were also analyzed at different developmental stages (Fig. 2b). Overall, plants of different transgenic lines showed comparable GUS staining patterns, but the intensity of the GUS staining differed amongst plants. In the very young seedlings (stage 0.7) no GUS staining was detected (Fig. 2c). In the cotyledons and 2-leaves stage (stages 1.00 and 1.02), plantlets showed a weak GUS activity spread over mesophyll cells. When plants developed (from stage 1.08 onwards), a very intense 


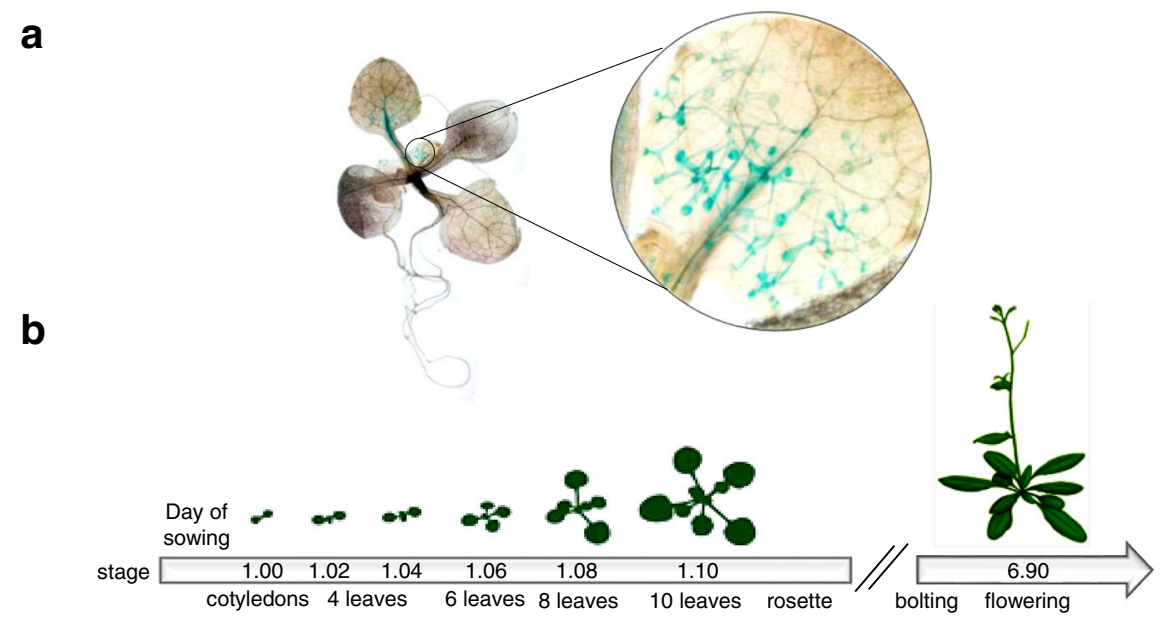

C
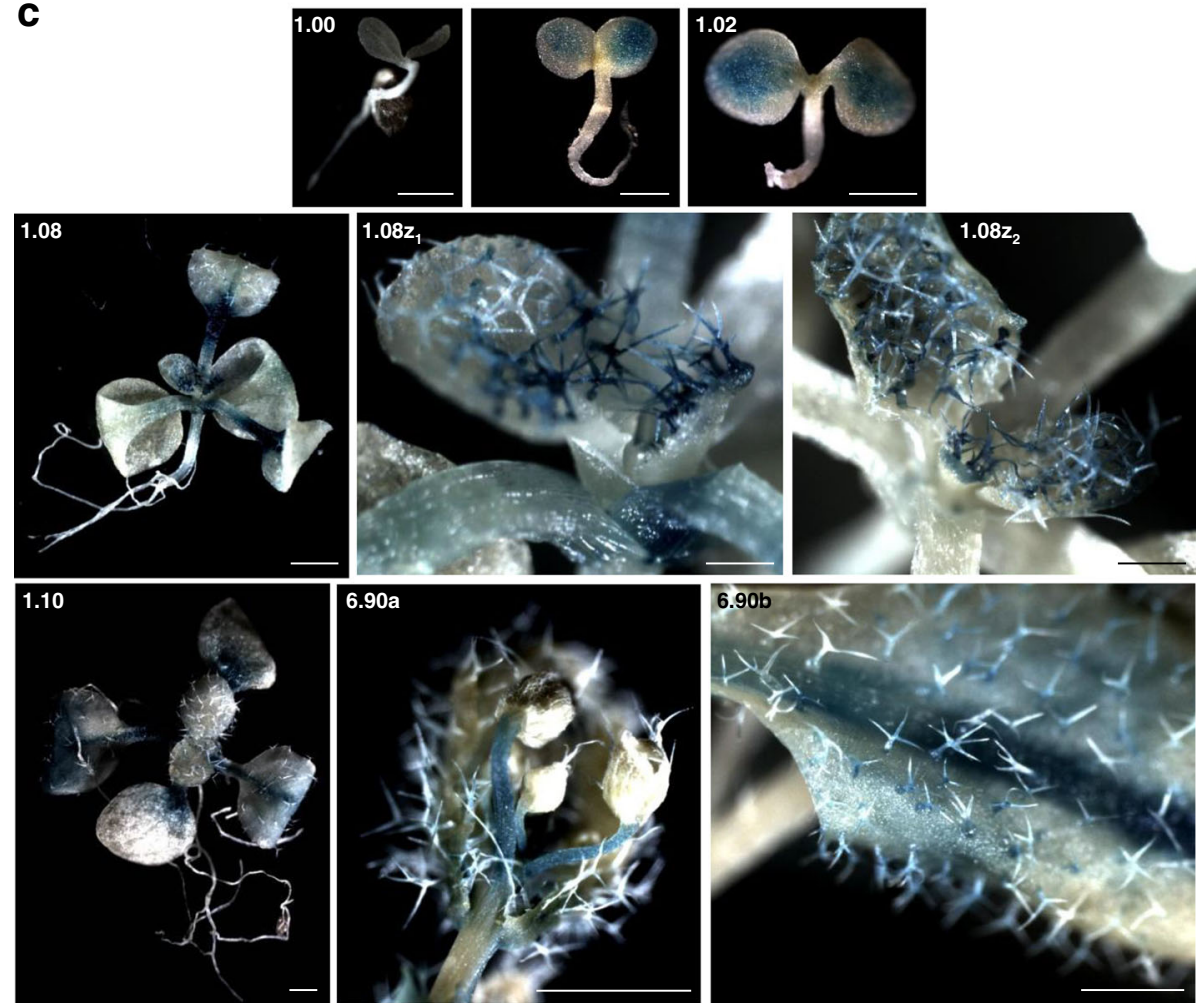

Fig. 2 Histochemical analysis of pAt2g02360:GUS A. thaliana lines. a, GUS staining data for 14-day-old plantlets. b, Different developmental plant stages analyzed [46]. c, GUS staining data for selected developmental stages with a closer look at trichomes present on new leaves (panel 1.08z) and on flower buds (panel 6.90a); panel 6.90b represents a close-up of rosette leaves. Scale bars represent $1 \mathrm{~mm}$

GUS activity was observed all over the petioles and in the majority of the trichomes present on the shoot meristem and first leaves. GUS staining in the trichomes present on newly developing leaves remained throughout further development of the plants. In plantlets of stage 1.10 and older (i.e., rosette stage), GUS staining was prominently visible in trichomes present on new leaves and in the petioles, the major leaf vein and some parts with mesophyll cells of older leaves. Trichomes present on older leaves also showed GUS staining, especially in those located in close proximity of the petiole, but their GUS staining was less intense compared to the staining detected in trichomes residing on young leaves. In flowering plants (stage 6.90), GUS staining was mainly visible in the flowers, some siliques and in some major veins and trichomes located in close proximity of these veins on rosette and cauline leaves. The CaMV35S:GUS control plants showed an intense blue, homogeneous GUS staining pattern in all organs and tissues of the plants throughout development (Results not shown). 
F-box-Nictaba is predominantly expressed in the trichomes of $A$. thaliana plants

Given the apparent activity of the pAt2g02360:GUS reporter construct in leaf trichomes and the occurrence of putative cis-regulatory elements reported to be responsible for trichome-specific gene regulation in the F-box-Nictaba promoter sequence including eight MYB-like recognition sites and five T/G-box elements (Additional file 1: Figure S5), the expression of the F-box-Nictaba gene was quantified by qRT-PCR in trichomes purified from WT $A$. thaliana Col-0 rosette leaves. At2g02360 mRNA levels measured in trichomes were slightly higher compared to those measured in unprocessed rosette leaves (containing trichomes) with increased expression in trichomes up to approximately 1.5-fold in 4-week-old plants and 2-fold in 3- and 5-week-old plants (Fig. 3a).

F-box-Nictaba gene expression was also analyzed in rosette leaves of mutant $A$. thaliana plants completely lacking trichomes. These GLABRA1 plants are impaired in the GL1 gene (At3g27920) which encodes a MYB-like transcription factor required for trichome development [25]. Since the GLABRA1 mutants are made in a Landsberg erecta-0 (Ler-0) background, F-box-Nictaba expression was also examined in WT A. thaliana Ler-0 plants. Trichome purification from rosette leaves of $A$. thaliana Ler-0 plants yielded similar amounts of trichomes as for A. thaliana Col-0 plants. However, the relative expression of the F-boxNictaba gene was much more pronounced in trichomes of A. thaliana Ler-0 plants with an up-regulation of 3.2-fold (although not statistically significant), 150-fold and 25-fold compared to total leaf tissue from 3-, 4- and 5-week-old $A$. thaliana Ler-0 plants, respectively (Fig. $3 \mathrm{~b}$, white bars). In comparison to GLABRA1 A. thaliana plants, At2g02360 transcript levels were 20-, 160- and 175-fold higher in trichomes from $A$. thaliana Ler-0 plants when compared to total leaf tissue from 3-, 4- and 5-week-old GLABRA1 $A$. thaliana plants, respectively (Fig. 3b, black bars).

The transcript levels for the F-box-Nictaba gene were higher in WT A. thaliana leaves of plants with a Col-0 background compared to leaves from WT plants with a Ler-0 background, at all developmental stages investigated, irrespective whether the purified trichomes or complete rosette leaves of both ecotypes were assessed (Fig. 3c). The differential expression was most striking when unprocessed rosette leaves were compared (gray bars): At2g02360 mRNA levels were approx. 160, 740 and 130 times higher in leaves of 3-, 4- and 5-week-old plants of Col-0 ecotype, respectively, compared to leaves of the same age from plants with a Ler-0 background. In the trichomes isolated from 3-, 4- and 5-week-old WT $A$. thaliana Col-0 plants F-box-Nictaba gene expression was 90-, 7- and 10-fold higher than in the trichomes of 3-, 4and 5-week-old plants from WT A. thaliana Ler-0, respectively (Fig. 3c, black bars).

\section{GALT1 and FUT13 genes are up-regulated in the tri- chomes of WT A. thaliana Ler-0 plants}

F-box-Nictaba was identified as a functional lectin that can bind $N$ - and $O$-glycans containing lactosamine
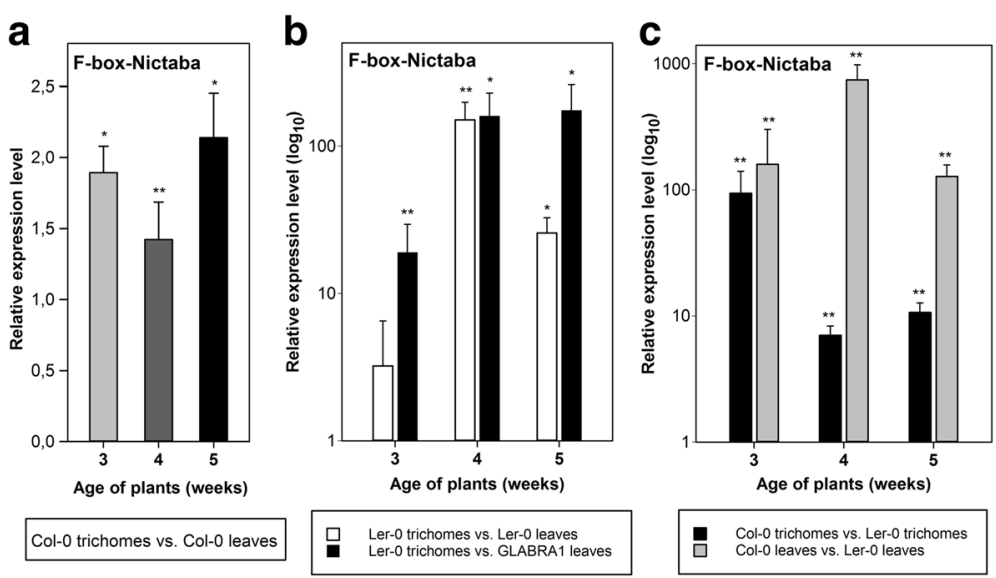

Fig. 3 Transcript levels of At2g02360 in the trichomes isolated from rosette leaves of WT A. thaliana plants of Col-0 or Ler-0 ecotype compared to corresponding gene expression levels in complete rosette leaves from WT A. thaliana plants of Col-0 or Ler-0 ecotype or of GLABRA1 A. thaliana plants. Relative transcript levels were determined in 3-, 4- and 5-week-old plants by qRT-PCR analyses of two independent biological experiments, each with two technical replicates; error bars \pm SE. Asterisks indicate statistically significant differential expression compared to control samples (complete rosette leaf material). a, gene expression levels in the trichomes of WT A. thaliana Col-0 plants compared to complete rosette leaves from WT A. thaliana Col-0 plants. $\mathbf{b}$, gene expression levels in the trichomes of WT A. thaliana Ler-0 plants compared to complete rosette leaves of WT A. thaliana Ler-0 plants or compared to complete rosette leaves of GLABRA1 A. thaliana plants as well as in complete rosette leaves of WT A. thaliana Ler-0 plants compared to complete rosette leaves of GLABRA1 plants. c, Comparison of gene expression levels for F-box-Nictaba in the trichomes and in complete rosette leaves of WT A. thaliana Col-0 compared to WT A. thaliana Ler-0 plants 
structures, Lewis a, Lewis $\mathrm{x}$ and Lewis y motifs [18]. Arabidopsis plants were only reported to contain Lewis a-modified $\mathrm{N}$-glycans. To our knowledge, the presence of Lewis a has not been studied yet in trichomes of $A$. thaliana plants. Since F-box-Nictaba expression is prominent in the trichomes, we addressed whether Lewis a motifs could be specifically synthesized in the trichomes. Therefore, qRT-PCR analysis was performed to analyse gene expression for the enzymes indispensable for Lewis a epitope synthesis in $A$. thaliana: i.e., At1g26810 encoding $\beta 1$,3-galactosyltransferase (GALT1) and At1g71990 encoding $\alpha 1,4$-fucosyltransferase (FUT13) (Fig. 4) [21, 24, 26]. According to Fig. 4, both GALT1 and FUT13 are expressed in rosette leaves as well as in the leaf trichomes of A. thaliana Col-0 plants. The transcript levels for GALT1 were significantly lower in the trichomes of WT A. thaliana Col-0 plants compared to those measured in the unprocessed rosette leaves of the same plants at all investigated developmental stages, the lowest value being a 5 -fold decrease of GALT1 expression in trichomes of 4-week-old plants (Fig. 4a). In 3- and 5-week-old plants GALT1 mRNA levels were approximately two and three times lower in trichomes compared to rosette leaves, respectively (Fig. 4a). In contrast to GALT1, FUT13 did not show a significant differential expression between trichomes and unprocessed rosette leaves (Fig. 4b).

Conversely, in trichomes of 3- and 4-week-old WT A. thaliana Ler-0 plants, the expression of the GALT1 gene was markedly up-regulated by 7 - and 29 -fold, respectively, when compared to levels in unprocessed rosette leaves originating from these plants (Fig. 4c, white bars). Comparison of the GALT1 expression levels in trichomes of WT A. thaliana Ler-0 plants to those in the trichomeless GLABRA1 leaves revealed that GALT1 mRNA levels are 5-, 20- and 7-fold higher in the trichomes, respectively, for the three plant stages tested (Fig. 4c, black bars). The differences in FUT13 expression levels between trichomes and unprocessed rosette leaves from WT A. thaliana Ler-0 plants were smaller than for the GALT1 gene expression. FUT13 expression in trichomes of 4-week-old plants was only 4 times higher, whereas in 3- and 5-week-old plants there was no apparent difference in FUT13 expression in trichome RNA and total RNA of Ler-0 leaf material (Fig. 4d, white bars). However, FUT13 expression was 2.5-fold upregulated in trichomes of $A$. thaliana Ler- 0 plants compared to GLABRA1 leaves of 4-week-old plants (Fig. 4d, black bars). Similarly to the F-box-Nictaba expression pattern, GALT1 and FUT13 genes are more significantly up-regulated in the trichomes of 4-week-old plants compared to those of 3- and 5-week-old plants.

The GALT1 gene was 3.5-7 times more expressed in the leaves of $A$. thaliana Col- 0 plants compared to the corresponding leaves of $A$. thaliana Ler-0 plants (Fig. 4e, gray bars), with the highest differential expression in 4-week-old plants. In contrast, GALT1 gene expression was 3-fold and 30-fold higher in the trichomes of 3- and 4-week-old $A$. thaliana Ler-0 plants compared to $A$. thaliana Col-0 plants (Fig. 4e, black bars). In general, WT A. thaliana Ler-0 plants had slightly higher transcript levels for FUT13 than the WT A. thaliana Col-0 plants in unprocessed rosette leaves (Fig. 4f, gray bars). More important differences were observed in the trichomes of 3- and 4-week-old plants, where the FUT13 transcript level was 2- and 5-fold higher in the A. thaliana plants with a Ler-0 background (Fig. 4f, black bars).

\section{Over-expression of F-box-Nictaba abrogates leaf damage and suppresses Pst DC3000 colonization at 4 and $5 \mathrm{dpi}$}

Since F-box-Nictaba gene expression was significantly up-regulated after SA treatment (Fig. 1b) and infection with Pst DC3000 (Fig. 1d), it was suggested that F-box-Nictaba plays a role in plant defense responses. To investigate the relevance of F-box-Nictaba for plant resistance towards stress, transgenic A. thaliana $\mathrm{Col}-0$ plants impaired in F-box-Nictaba gene expression (KO6 line) as well as plants with increased F-box-Nictaba gene expression (OE lines) (Additional file 1: Text S2) were subjected to infection with the virulent Pst DC3000 strain and assessed for disease symptoms and bacterial growth. The level of colonization $24 \mathrm{~h}$ post infection reflects the initial growth of Pst DC3000 within the apoplast, whereas growth of the pathogen in the next days after infection is accompanied by host programmed cell death and necrosis. Beforehand, transgenic F-boxNictaba plants were proven to be morphologically indistinguishable from WT plants throughout growth and development (Additional file 1: Text S2).

As demonstrated in Fig. 5a and in Additional file 1: Figure S6, KO6 plants developed similar disease symptoms to WT plants, characterized by leaf lesions constituting up to 30 and $35 \%$ of total leaf area at 4 and 5 dpi, respectively. Plants of both OE lines clearly exhibited less disease symptoms than the WT plants at 4 and $5 \mathrm{dpi}$, with lesions covering 8 to $12 \%$ of total leaf area. Also at $3 \mathrm{dpi}$, OE4 plants demonstrated reduced disease symptoms compared to the WT plants. Consistent with the reduced levels of leaf damage, there was no significant difference in colonization of WT and KO6 plants whereas both OE lines yielded significantly lower bacterial counts compared to WT samples at $4 \mathrm{dpi}$ and at $5 \mathrm{dpi}$ (Fig. 5b).

qRT-PCR analysis demonstrated no differential expression for any of the two positive controls WRKY7O and PR1, neither in infected and mock-treated KO6 plants (Fig. 6a-b) nor in OE4 and OE6 plants (Fig. 6d-e). F-boxNictaba expression remained stable in all transgenic 

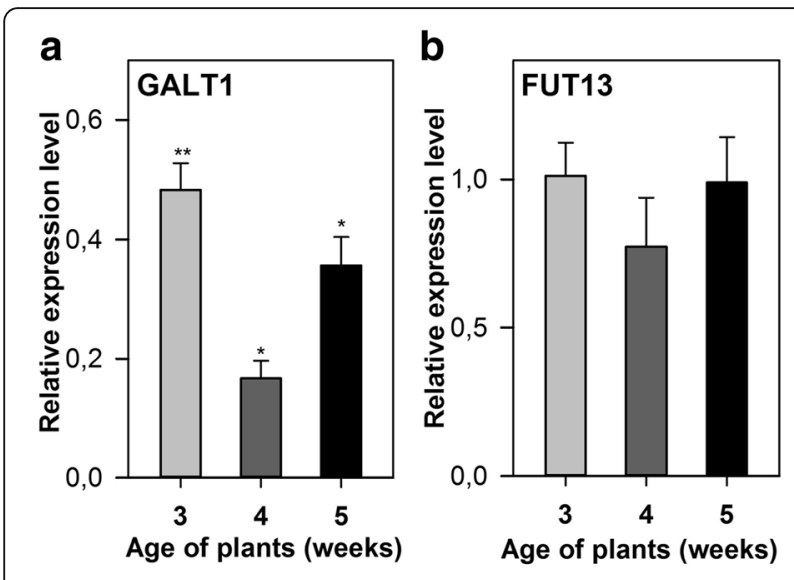

Col-0 trichomes vs. Col-0 leaves

C

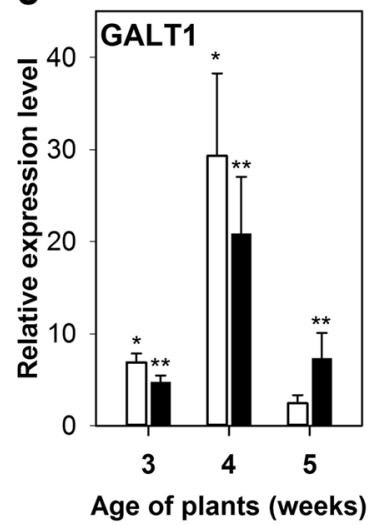

d

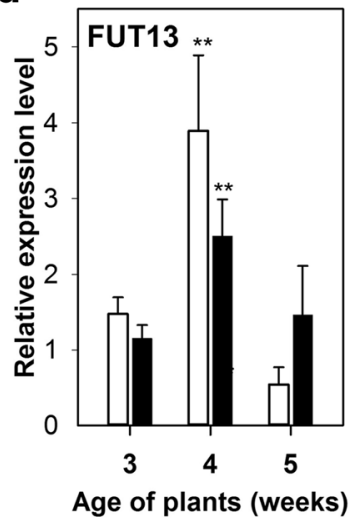

Ler-0 trichomes vs. Ler-0 leaves Ler-0 trichomes vs. GLABRA1 leaves

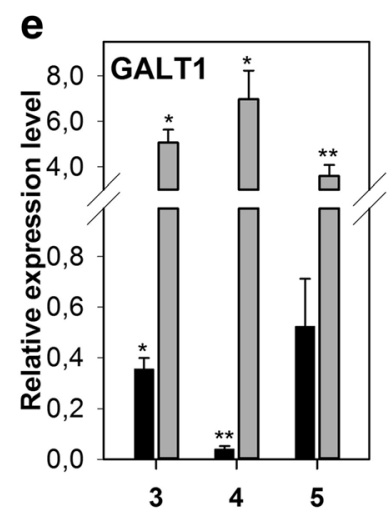

Age of plants (weeks)

\section{f}

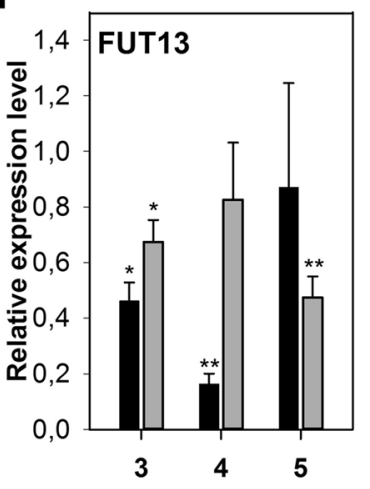

Age of plants (weeks)
Col-0 trichomes vs. Ler-0 trichomes Col-0 leaves vs. Ler- 0 leaves
Fig. 4 Transcript levels of GALT1 (At1g26810) and FUT13 (At1g71990) in trichomes isolated from rosette leaves of WT A. thaliana plants of Col-0 or Ler-0 ecotype compared to corresponding gene expression levels in complete rosette leaves of WT A. thaliana plants of Col-0 or Ler-0 ecotype or of GLABRA1 A. thaliana plants. Transcript levels were determined in 3-, 4- and 5-week-old plants by qRT-PCR analyses of two independent biological experiments, each with two technical replicates; error bars \pm SE. Asterisks indicate statistically significant differential expression compared to control samples (complete rosette leaf material). a-b, Relative gene expression levels of GALT1 (a) and FUT13 (b) in the trichomes of WT A. thaliana Col-0 plants compared to complete rosette leaves from WT A. thaliana Col-0 plants. c-d, Relative gene expression levels of GALT1 (c) and FUT13 (d) in the trichomes of WT A. thaliana Ler-0 plants compared to complete rosette leaves of WT A. thaliana Ler-0 plants or compared to complete rosette leaves of GLABRA1 A. thaliana plants as well as in complete rosette leaves of WT A. thaliana Ler-0 plants compared to complete rosette leaves of GLABRA1 plants. e-f, Comparison of gene expression levels of GALT1 (e) and FUT13 (f) in the trichomes and in complete rosette leaves of WT A. thaliana Col-0 plants compared to WT A. thaliana Ler-0 plants

plants over the time course of the infection experiment, with very low transcript levels in the $\mathrm{KO}$ line and significant 100-200-fold over-expression in the two OE lines compared to WT plants (Fig. 6c and f).

\section{Discussion}

The qRT-PCR data and the meta-analysis database search show that, although a basal expression of At2g02360 is present throughout plant development, this F-box-Nictaba is a SA- and pathogen-inducible gene. Supported by the expression profile of the WRKY7O gene and the promoter analysis of At2g02360, it is likely that the SA-inducible expression of $A t 2 g 02360$ is under the control of (a) SA-dependent WRKY transcription regulator(s). Judging from the delay in maximal expression for the F-box-Nictaba gene in comparison to one of the key SA-dependent WRKY transcription factors it is suggested that F-box-Nictaba is a late SA-responsive gene. As demonstrated by the Pseudomonas infection assays, At2g02360 expression does not play an essential role in pathogen defense. It was reported previously that the A. thaliana genome contains more than 20 homologous genes encoding At2g02360-like genes for which evidence of expression is available [17, 19]. All these genes show considerable amino acid sequence similarity, reaching levels of $>90$ and $>40 \%$ sequence similarity for the F-box domain and the Nictaba domain, respectively. It is likely that some of these genes show functional redundancy and could fulfill the same or at least a similar role, as also demonstrated during male gametogenesis [27]. Therefore, it is suggested that At2g02360 will rather act as a partner in a complex network of defense-related proteins. Since transcript levels for F-box-Nictaba were up-regulated in WT $A$. thaliana plants after 

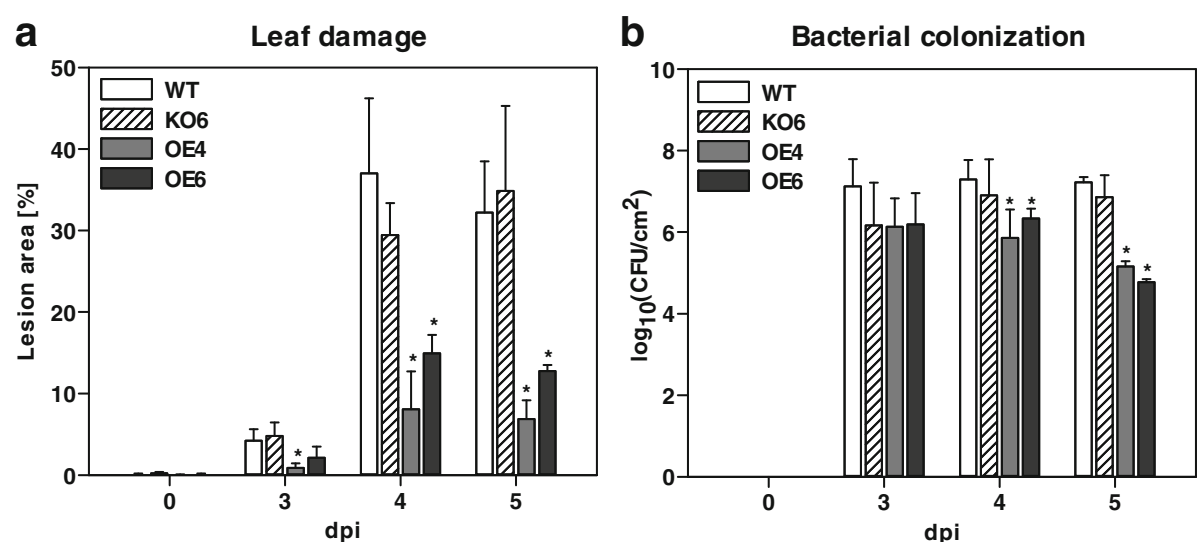

Fig. 5 Development of disease symptoms and pathogen growth on the leaves of WT and transgenic KO6, OE4 and OE6 A. thaliana plants infected with Pst DC3000. a, Disease symptoms on leaves of infected A. thaliana plants measured as percent of lesion area. $\mathbf{b}$, Pathogen growth in infected leaves. Levels of colonization are scored as CFU cm${ }^{-2}$. The analysis was performed for two independent biological experiments, each with two technical replicates; error bars $\pm \mathrm{SE} ;{ }^{*} p<0.05 ;{ }^{* *} p<0.01$

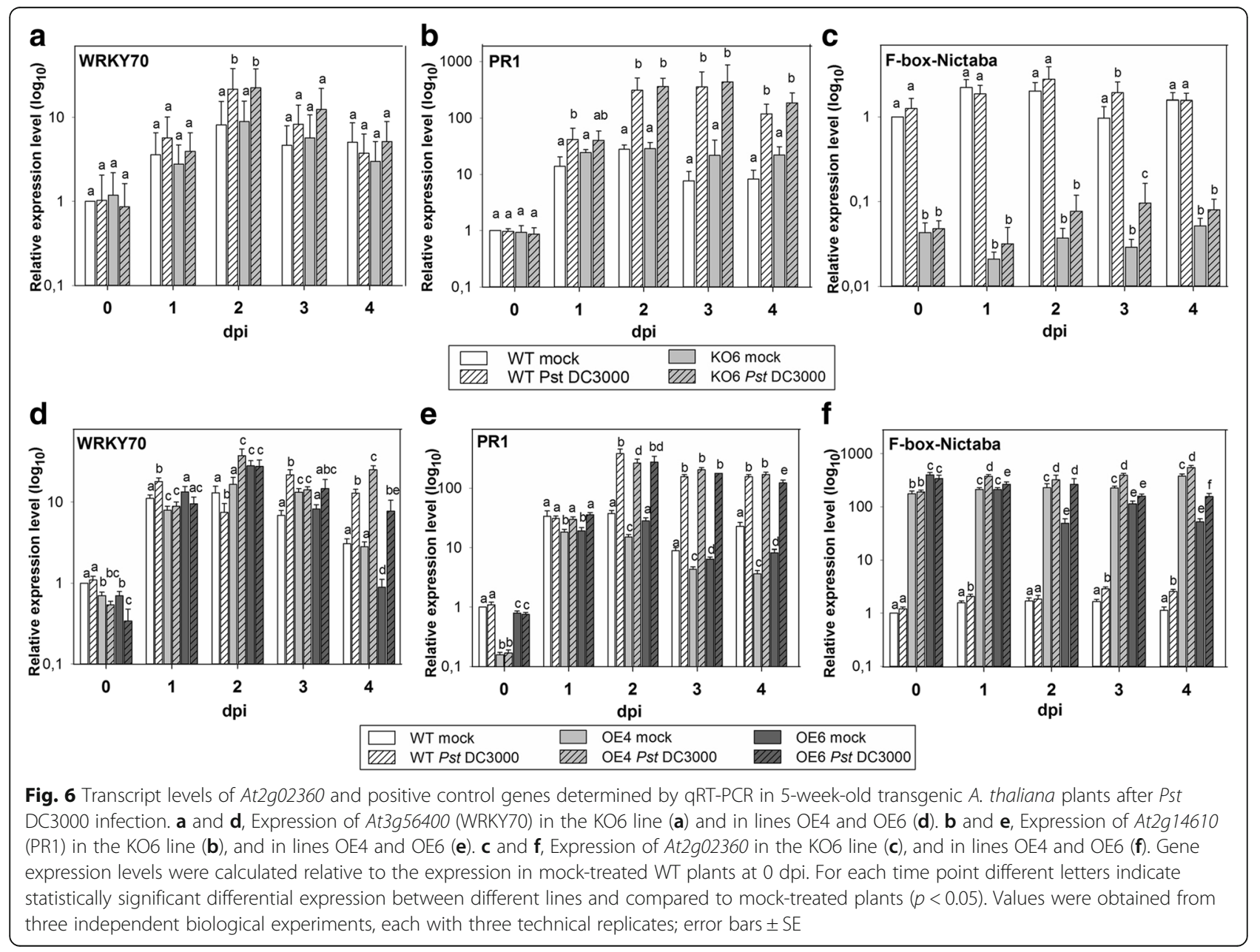


SA treatment and Pst DC3000 infection but expression was not affected by MeJA/ethephon treatment or infection with $B$. cinerea, it is reasonable to conclude that Fbox-Nictaba plays a role in SA-mediated plant defense reactions to pathogen infection.

F-box-Nictaba expression is not only responsive towards biotic stresses, it is also up-regulated up to 3-fold after heat stress. Cross-talk between biotic and abiotic stress signaling pathways is quite common [28, 29]. F-box-Nictaba up-regulation upon heat stress might be driven by heat shock-activated transcription factors which can bind to the heat shock-responsive cis-elements present in the At2g02360 promoter sequence, but also by heat stress-induced WRKY transcription factors activating F-box-Nictaba expression through the multiple W-box motifs present in its promoter sequence. Expression analysis of the F-box-Nictaba gene in WRKY-specific mutant lines could clarify the mode of transcriptional regulation of F-box-Nictaba.

In line with the plant defense-related expression of At2g02360, the histochemical assays showed preferential activity of the At $2 g 02360$ promoter throughout plant development in non-glandular leaf trichomes of transgenic A. thaliana plants, especially in the trichomes present on young leaves. Similarly the qRT-PCR assays demonstrated a dominant presence of At $2 g 02360$ mRNA in Arabidopsis leaf trichomes. Non-glandular trichomes are epidermal hairs on the surface of most plant aerial organs, characterized by a unicellular dendritic structure with stalk and three to four branches. They are implicated in transpiration control, thermotolerance and protection against insects, diseases and UV irradiation [30]. Although non-glandular trichomes are presumably non-secreting, they do express genes involved in the biosynthesis of secondary compounds suggesting their putative role in plant defense against pathogens [31]. In line with the presumed WRKY-dependent regulation of F-box-Nictaba, WRKY transcription factors are highly expressed in trichome tissues [32]. Alternatively, F-box-Nictaba gene expression in the trichomes may be under control of MYB transcription factors [33], which could bind to the multiple MYB-like cis-regulatory elements present in the F-box-Nictaba promoter and are reported to be responsible for trichome-specific gene regulation [34, 35]. Interestingly, two other F-box-Nictaba homologs are also predominantly expressed in trichomes [4]. One of these homologs, encoded by At1g80110, is up-regulated both in the trichomes and after infection with Pst DC3000.

The higher transcript levels for both GALT1 and FUT13 in the trichomes of WT A. thaliana with Ler-0 background strongly resembled the trichome-specific expression of the F-box-Nictaba gene under study. In general, all three genes are up-regulated more significantly in the trichomes of 4-week-old A. thaliana Ler-0 plants compared to those of
3- and 5-week-old plants. It is not entirely clear why the highest trichome-specific expression was reached at this particular time point, but the most probable explanation is that 4-week old $A$. thaliana plants are undergoing transition from the vegetative to the reproductive phase, characterized by stem formation and progression into bolting [36]. This is the time when major physiological changes are likely to occur and trichome production was shown to be affected significantly during this process [37-39]. Irrespective of the cause of the observed time-dependent differences, these data indicate that the two enzymes GALT1 and FUT13, required for biosynthesis of Lewis a structures, and the carbohydrate-binding protein F-box-Nictaba are co-expressed in the trichomes of $A$. thaliana Ler- 0 plants. The fact that Lewis a containing glycans can be synthesized in the trichomes does not indicate that they are available to Fbox-Nictaba within the nucleocytoplasmic compartment of the cell. Future studies will include interaction studies to clarify (1) the availability of Lewis a modified proteins in leaf trichomes and (2) the binding of the SA-induced F-box-Nictaba protein to these glycan structures.

\section{Conclusions}

Detailed analysis of the transcript levels for the carbohydrate-binding F-box-Nictaba protein from A. thaliana demonstrated up-regulation after plant treatment with stress stimuli of both biotic and abiotic origin, including heat stress, the defense related plant hormone salicylic acid and after plant infection with the virulent Pseudomonas syringae pv. tomato strain DC3000. Furthermore, the histochemical assays show that the F-box-Nictaba gene is abundantly expressed in leaf trichomes, appendages involved in plant defense responses. Experiments with transgenic A. thaliana plants overexpressing the F-boxNictaba protein demonstrated reduced disease symptoms after Pst DC3000 infection compared to the wild-type plants or plants in which F-box-Nictaba expression is reduced. Altogether the data strongly suggest that F-boxNictaba is a defense protein involved in the plant response against Pseudomonas infection. Furthermore, we show that both F-box-Nictaba as well as the glycosyltransferases required for the synthesis of glycan motifs specifically recognized by F-box-Nictaba are co-expressed in the trichomes. Although the putative targets of F-box-Nictaba and the underlying mechanism of action remain to be elucidated, our research sheds a new light on the putative function of the non-glandular trichomes in plant responses to pathogen attack through glycan-based stress signaling.

\section{Methods}

Plant materials and culture conditions

Seeds of WT A. thaliana ecotype Col-0 were purchased from Lehle Seeds (Round Rock, Texas, USA). Seeds of WT A. thaliana ecotype Ler-0 (NW20), GLABRA1 
(N64) and SALK_085735C (herein renamed line KO6) [40] were obtained from the European Arabidopsis Stock Centre (NASC, University of Nottingham, UK). To establish in vitro cultures, surface sterilized seeds were sown on sterile filter paper which was placed on top of solid Murashige and Skoog (MS) medium (Duchefa, Haarlem, The Netherlands). To establish in vivo cultures, surface sterilized seeds were directly sown into artificial soil (Jiffy-7, $44 \mathrm{~mm} \varnothing$ ) (AS Jiffy Products, Drobak, Norway), into expanded clay granules $(>4 \mathrm{~mm}$ $\varnothing)$ or in pot soil. To break dormancy, the seeds were stratified at $4{ }^{\circ} \mathrm{C}$ for three days in the dark. Afterwards, seeds were transferred to a controlled growth room set at $21{ }^{\circ} \mathrm{C}$ with a $16 / 8 \mathrm{~h}$ photoperiod for seed germination and plant development. Only for infection experiments and trichome isolation, seeds were kept in a growth chamber (Conviron Germany $\mathrm{GmbH}$, Berlin, Germany) set at $21{ }^{\circ} \mathrm{C}$ with a $12 / 12 \mathrm{~h}$ photoperiod. All seedlings and plants were watered regularly. Plant samples were collected from different developmental stages as defined by [41]. Early stage plant materials (cotyledons, 4-leaves and 8-leaves stage) were collected from in vitro grown plants and included the complete seedlings, whereas the other samples were taken from plants grown in artificial soil. Root material was collected from plants grown in granules. Trichomes were isolated from rosette leaf material collected from plants grown in pot soil.

\section{Chemical reagents}

BAP, $\mathrm{GA}_{3}$ and MeJA were purchased from Sigma-Aldrich (Bornem, Belgium). ABA and ethephon were obtained from Acros Organics (Geel, Belgium). SA, indole acetic acid (IAA) and salt were obtained from Duchefa, whereas mannitol was purchased from VWR (Leuven, Belgium). MG132 was obtained from Enzo Life Sciences (Antwerpen, Belgium). Prior to use, appropriate amounts of MeJA, SA, $\mathrm{ABA}, \mathrm{IAA}$ and $\mathrm{GA}_{3}$ were dissolved in $100 \%(\mathrm{w} / \mathrm{v})$ ethanol, whereas BAP and MG132 were dissolved in $100 \%(\mathrm{w} / \mathrm{v})$ dimethyl sulfoxide (DMSO) (VWR). Ethephon, $\mathrm{NaCl}$ and mannitol were dissolved in water.

\section{Hormone treatments, abiotic stress applications and infection assays on WT $A$. thaliana plants}

Hormone and abiotic stress treatments were performed according to [42-46] with modifications. Namely, in vitro grown 16-day-old seedlings were transferred from MS agar plates to Petri dishes filled with liquid MS medium containing either a hormone solution $(100 \mu \mathrm{M}$ concentration in case of ABA, BAP, ethephon, $\mathrm{GA}_{3}$, IAA or MeJA and $300 \mu \mathrm{M}$ concentration in case of $\mathrm{SA}$ ), $50 \mu \mathrm{M}$ MG132, $150 \mathrm{mM} \mathrm{NaCl}$ or $100 \mathrm{mM}$ mannitol and incubated at $21{ }^{\circ} \mathrm{C}$ for appropriate times. Controls were kept on liquid MS medium containing an equal volume of the corresponding solvent (ethanol, DMSO or water).
Cold and heat stress were applied by incubating the MS agar plates with the seedlings in the dark either at $4{ }^{\circ} \mathrm{C}$ or $37{ }^{\circ} \mathrm{C}$, respectively. Concomitantly, controls were incubated at $21{ }^{\circ} \mathrm{C}$ in the dark. For every stress application 20-30 seedlings were collected for RNA extraction at several time points, immediately frozen in liquid nitrogen and stored at $-80{ }^{\circ} \mathrm{C}$ until use. Infection assays with Pseudomonas syringae pv. tomato strain DC3000 and Botrytis cinerea strain B05.10 were performed as described elsewhere [47-49]. For each infection, one hundred individually grown 5-week-old Arabidopsis plants were inoculated, either with infection or mock solution. Infection with $P$. syringae was performed by spraying the rosette leaves until run-off with bacteria from the mid to late log phase of growth $\left(\mathrm{OD}_{600}=\right.$ 0.6-1.0), The inoculation solution consisted of bacteria resuspended in $10 \mathrm{mM} \mathrm{MgSO}_{4}$ supplemented with $0.05 \%$ Silwet-77 (GE Specialty Materials (Suisse) S.a.r.l., Switzerland) to obtain a bacterial solution of $\mathrm{OD}_{600}=$ 0.05 corresponding to $2.5 \times 10^{7} \mathrm{cfu} / \mathrm{ml}$. One day before treatment up till two days after bacterial infection, the plants were maintained at $100 \%$ relative humidity to increase the infection efficiency. Infection with $B$. cinerea was done by the droplet technique with spores harvested from 10-day-old cultures by putting a 10- $\mu$ l droplet of spore solution on the upper side of three randomly chosen rosette leaves from each plant. The inoculation solution contained $5 \times 10^{5}$ conidia / $\mathrm{ml}$ in $1 / 2$ strength potato dextrose broth medium. In the fungal assay, the plants were kept at $100 \%$ relative humidity during the entire experiment. At indicated time points post each infection, rosette leaves of 8-10 randomly chosen plants were frozen in liquid nitrogen and stored at $-80{ }^{\circ} \mathrm{C}$ prior to use. All experiments for each hormone treatment, abiotic stress application and infection assay with $P$. syringae were performed with two independent biological experiments, and three technical replicates for each analysis. The infection experiment with $B$. cinerea was performed with two independent biological experiments, and two technical replicates.

\section{RNA extraction and cDNA synthesis}

Plant samples were ground into a fine powder with a mortar and pestle and RNA extracted using TRI reagent (Sigma-Aldrich) according to the manufacturer's instructions. To remove any residual genomic DNA, samples were treated with 2 units of RNase-free DNaseI (Fermentas, St. Leon-Rot, Germany) for $30 \mathrm{~min}$ at $37^{\circ} \mathrm{C}$. After addition of $2 \mu \mathrm{l}$ EDTA $(25 \mathrm{mM})$, the DNase enzyme was inactivated by incubation at $65{ }^{\circ} \mathrm{C}$ for $10 \mathrm{~min}$. The RNA concentration and purity were measured with a Nanodrop 2000 Spectrophotometer (Thermo Scientific, USA). First-strand cDNA was synthesized from $1 \mu \mathrm{g}$ of DNA-free total RNA with $1 \mu \mathrm{L}$ 
of $50 \mu \mathrm{M}$ oligo $(\mathrm{dT})_{20}$ using the M-MLV transcriptase kit (Invitrogen) and then diluted $2.5 \mathrm{x}$ with RNase-free water. cDNA quality was checked by RT-PCR using primers specific for the UBC9 gene (Additional file 1: Table S2).

\section{Quantitative RT-PCR assay}

qRT-PCR analyses were performed using the SensiMix SYBR kit (Bioline Reagents Ltd, London, UK). The reaction mixture contained $1 \mathrm{x}$ SensiMix ${ }^{\mathrm{mm}}$ SYBR, 2 ng $\mu \mathrm{L}^{-1}$ firststrand cDNA and $500 \mathrm{nM}$ of gene-specific forward and reverse primers (Additional file 1: Table S2) in a total volume of $20 \mu \mathrm{L}$. For hormone and stress treatments specific positive control genes were included according to [50-54]. qRT-PCR was carried out with 3 technical replicates in a Rotor-Gene 3000 (Corbett Life Science) using Rotor Discs (Qiagen, Hilden, Germany) as described [55].

\section{Construction of vectors for the GUS reporter system and for over-expression of F-box-Nictaba}

The pAt2g02360:GUS reporter construct as well as the CaMV 35S:At2g02360 construct were generated using the Gateway ${ }^{\mathrm{TM}}$ cloning technology (Invitrogen). A $1806 \mathrm{nt}$ At $2 g 02360$ promoter fragment (including the 5' UTR) was amplified by a two-step PCR starting from total genomic DNA extracted from WT A. thaliana Col-0 plants. The At2g02360 coding sequence was amplified by a two-step PCR starting from the cDNA clone BX820545 (INRA, Centre de Toulouse, Unité de Recherche 1258-CNRGV, Castanet-Tolosan Cedex, France). In the first step, primers were used to generate an At2g02360 promoter sequence (primers evd555 and evd556) and an At2g02360 gene sequence (primers evd1046 and evd1047) including parts of the attB1 and attB2 Gateway adaptor sites at their $5^{\prime}$ and 3' sequences, respectively (Additional file 1: Table S3). Primer sequences for promoter amplification were made based on At2g02360 gene information as available on TAIR10. In the second step, primers evd2 and evd4 were used to complete the attB sites (Additional file 1: Table S3). AttB-PCR products were then cloned via the pDONR221 donor vector (Invitrogen) into the pKGWFS7.0 or pK7WG2.0 destination vector for promoter and gene sequence, respectively [56], sequenced and introduced into $A$. tumefaciens strain GV3101 using electroporation. WT Arabidopsis Col-0 plants were transformed using the floral-dip method [57]. Transgenic progenies were selected on MS agar plates supplemented with $75 \mathrm{mg} \mathrm{L}^{-1}$ kanamycin. Integration of the T-DNA into the plant genome was checked by PCR on genomic DNA using GUS-specific primers GUS-F and GUS-RV or kanamycin-specific primers evd463 and evd261 (Additional file 1: Table S3). Plants homozygous for the promoter:GUS construct in the T3 generation were used for the histochemical assays. Plants homozygous for the CaMV 35S:At2g02360 construct (OE lines) in the T4 generation were used for infection experiments.

\section{Histochemical GUS assays}

The GUS assay was performed according to [58]. Microscopic analysis was performed on a Nikon eclipse TE2000-e microscope (Nikon Belux, Brussels, Belgium) and a Leica DFC400 microscope (Leica, Heerbrugg, Germany) using the NIS-Elements and Leica Application Suite software packages, respectively.

\section{Isolation of leaf trichomes and subsequent RNA analysis}

Leaf trichomes were isolated from rosette leaves of 3-, 4and 5-week-old plants according to [59]. Purified trichomes were frozen in liquid nitrogen and stored at $-80{ }^{\circ} \mathrm{C}$ until further processing. Yield, purity and integrity of the isolated trichomes was assessed by transmission microscopy using a Leica DFC400 microscope (Leica) with the Leica Application Suite software packages. For total RNA extraction, $1 \mathrm{~mL}$ TRI Reagent (Sigma-Aldrich) and $50 \mathrm{mg}$ glass beads $(300 \mu \mathrm{m} \varnothing$, Sigma-Aldrich) were added to approx. 60,000-80,000 trichomes (isolated from $25 \mathrm{~g}$ of rosette leaves). The solution was mixed at max speed (five cycles of $20 \mathrm{~s}$ mixing and $20 \mathrm{~s}$ rest on ice). For RNA extraction from unprocessed rosette leaves, $1 \mathrm{~mL}$ TRI Reagent was added to approx. $100 \mathrm{mg}$ samples previously ground to a fine powder with a mortar and pestle. Trichome and complete leaf tissue extracts were then processed according to the manufacturer's recommendations. DNase treatment, cDNA synthesis, RT-PCR and qRT-PCR were performed as described above. The complete experiment was repeated twice.

\section{Stress treatments of transgenic $A$. thaliana plants}

Approximately sixty individually grown 5 -week-old WT plants as well as transgenic F-box-Nictaba-specific KO and $\mathrm{OE}$ plants were inoculated with either the Pst DC3000 infection or the mock solutions as described above. At 0, 3, 4 and 5 dpi approximately hundred leaves were collected for each line and scanned with a flatbed scanner (Canoscan Lide 25). Scans of single leaves were processed with the ImageJ software. The damage to the leaves was determined as percent ratio of lesion area relative to the total leaf area using the disease assessment software of the APS Assess 2.0 program. Bacterial growth in the leaves of mock-treated and infected plants was determined by serial dilution assays as described [49]. Bacterial colonization was determined as colony forming units (CFU) $\mathrm{cm}^{-2}$ of sampled leaf tissue. RNA extraction, DNase treatment, cDNA synthesis and qRT-PCR for expression analysis at $0,1,2,3,4$ and 5 dpi were performed as described above. The whole experiment for leaf damage assessment and bacterial colonization was performed with two independent biological experiments and three technical replicates for the each analysis. 


\section{Additional file}

Additional file 1: Figure S1. At2g02360 promoter sequence, with major putative cis-acting regulatory elements highlighted. Figure S2. Level of At2g02360 expression during the lifecycle of WT A. thaliana Col-0 plants using the Genevestigator search tool. Figure S3. Relative transcript levels of selected positive control genes in stress-treated WT A. thaliana Col-0 plants. Figure S4. Relative transcript levels of At2g02360 in 16-day-old WT A. thaliana Col-0 seedlings different stress treatments. Figure S5. At2g02360 promoter sequence, with highlighted putative cis-acting regulatory elements possibly involved in promoter activity in trichomes. Figure S6. Development of disease symptoms on the leaves of WT and transgenic KO6, OE4 and OE6 A. thaliana plants infected with Pst DC3000. Table S1. Putative cis-acting regulatory elements identified with high frequency in the At2g02360 promoter sequence by in silico analyses for identical motifs stored in the PLACE, PlantCARE and AGRIS databases. Table S2. Overview of all primers used in qRT-PCR. Table S3. Overview of all primers used in molecular cloning. Text S1. In silico expression analysis indicates that At2g02360 is a stress-responsive gene. Text S2. Characterization of transgenic $A$. thaliana plants with altered F-boxNictaba expression. (DOCX $1858 \mathrm{~kb}$ )

\section{Abbreviations}

Dpi: Days post infection; ET: Ethylene; FBG: Glycan-binding F-box protein; FUT13: a1,4-fucosyltransferase; Gal: Galactose; GALT1: $\beta 1,3-$ galactosyltransferase; Glc: Glucose; GlcNAc: N-acetylglucosamine; JA: Jasmonic acid; KO: Knock-out; Le a: Lewis a structure; Nictaba: Nicotiana tabacum agglutinin; OE: Over-expression; Pst DC3000: Pseudomonas syringae pv. tomato strain DC3000; qRT-PCR: Quantitative reverse transcriptase polymerase chain reaction; SA: Salicylic acid

\section{Acknowledgements}

We thank Prof. dr. G. Gheysen (Dept. Molecular Biotechnology, Ghent University, Belgium) for providing the pCa35S:GUS Arabidopsis seeds and dr. D. De Vleesschauwer and Prof. dr. M. Höfte (Dept. Crop Protection, Ghent University, Belgium) for providing the pathogens and for technical assistance with infection assays. We acknowledge Prof. dr. D. Marks for technical advice on the trichome extraction protocol.

\section{Funding}

This research was funded by the Research Council of Ghent University (project BOF2010/GOA/003) and the Fund for Scientific Research-Flanders (FWO Grant G006114N). N.L. acknowledges the receipt of a postdoctoral fellowship from the Fund for Scientific Research-Flanders.

\section{Availability of data and materials}

The dataset(s) supporting the conclusions of this article is (are) included within the article and its additional files.

\section{Authors' contributions}

KS, NL and EJMVD conceived and designed the experiments. KS, NL, YZ, LE, JVH and BA performed the experiments. KS, NL and EJMVD analyzed the data and wrote the manuscript. All authors read and approved the final manuscript.

\section{Competing interests}

The authors declare that they have no competing interests.

\section{Consent for publication}

Not applicable.

\section{Ethics approval and consent to participate}

Not applicable.

Received: 16 March 2016 Accepted: 26 September 2016

Published online: 04 October 2016

\section{References}

1. Hua Z, Zou C, Shiu S-H, Vierstra RD. Phylogenetic comparison of F-box (FBX) gene superfamily within the plant kingdom reveals divergent evolutionary histories indicative of genomic drift. PLOS ONE. 2011;6:e0016219.
2. Petroski MD, Deshaies RJ. Function and regulation of cullin-RING ubiquitin ligases. Nat Rev Mol Cell Biol. 2005;6:9-20.

3. Skaar JR, Pagan JK, Pagano M. Mechanisms and function of substrate recruitment by F-box proteins. Nat Rev Mol Cell Biol. 2013;14:369-81.

4. Stefanowicz K, Lannoo N, Van Damme EJM. Plant F-box proteins - judges between life and death. Crit Rev Plant Sci. 2015:34:523-52.

5. Kelley DR, Estelle M. Ubiquitin-mediated control of plant hormone signaling Plant Physiol. 2012;160:47-55.

6. Somers DE, Kim W-Y, Geng R. The F-box protein ZEITLUPE confers dosagedependent control on the circadian clock, photomorphogenesis, and flowering time. Plant Cell. 2004;16:769-82.

7. Ito S, Song YH, Imaizumi T. LOv domain-containing F-box proteins: lightdependent protein degradation modules in Arabidopsis. Mol Plant. 2012;5:573-82.

8. Woo HR, Chung KM, Park JH, Oh SA, Ahn T, Hong SH, Jang SK, Nam HG ORE9, an F-box protein that regulates leaf senescence in Arabidopsis. Plant Cell. 2001:13:1779-90.

9. Kubo K, Entani T, Takara A, Wang N, Fields AM, Hua Z, Toyoda M, Kawashima S, Ando T, Isogai A, Kao TH, Takayama S. Collaborative non-self recognition system in S-Rnase-based self-incompatibility. Science. 2010;330:769-99.

10. Maldonado-Calderón MT, Sepúlveda-García E, Rocha-Sosa M. Characterization of novel F-box proteins in plants induced by biotic and abiotic stress. Plant Sci. 2012;185:208-17.

11. Marino D, Peeters N, Rivas S. Ubiquitination during plant immune signaling. Plant Physiol. 2012;160:15-27.

12. Guo LQ, Nezames CD, Sheng LX, Deng XW, Wei N. Cullin-RING ubiquitin ligase family in plant abiotic stress pathways. J Integr Plant Biol. 2013;55:21-30.

13. Yoshida Y, Chiba T, Tokunaga F, Kawasakik H, Iwai K, Suzuki T, Ito Y, Matsuoka K, Yoshida Y, Tanaka K. Lectin-like ERAD players in ER and cytosol. Biochim Biophys Acta. 1800;2010:172-80.

14. Glenn KA, Nelson RF, Wen HM, Mallinger AJ, Paulson HL. Diversity in tissue expression, substrate binding, and SCF complex formation for a lectin family of ubiquitin ligases. J Biol Chem. 2008;283:12717-29.

15. Yoshida Y, Adachi E, Fukiya K, Iwai K, Tanaka K. Glycoprotein-specific ubiquitin ligases recognize $\mathrm{N}$-glycans in unfolded substrates. EMBO Rep. 2005;6:239-44.

16. Hoseki J, Ushioda R, Nagata K. Mechanism and components of endoplasmic reticulum-associated degradation. J Biochem. 2010;147:19-25.

17. Lannoo N, Peumans WJ, Van Damme EJM. Do F-box-proteins with a Cterminal domain homologous with the tobacco lectin play a role in protein degradation in plants? Biochem Soc Trans. 2008;36:843-7.

18. Stefanowicz K, Lannoo N, Proost P, Van Damme EJM. Arabidopsis F-box protein containing a Nictaba-related lectin domain interacts with $\mathrm{N}$ acetyllactosamine structures. FEBS Open Bio. 2012;2:151-8.

19. Delporte A, Van Holle S, Lannoo N, Van Damme EJM. The tobacco lectin, prototype of the family of Nictaba-related proteins. Curr Protein Pept Sci. 2015;16:5-16.

20. Lannoo N, Peumans WJ, Van Pamel E, Alvarez R, Xiong TC, Hause G, Mazars C, Van Damme EJM. Localization and in vitro binding studies suggest that the cytoplasmic/nuclear tobacco lectin can interact in situ with highmannose and complex N-glycans. FEBS Lett. 2006:580:6329-37.

21. Léonard R, Costal G, Darrambide E, Lhernould S, Fleurat-Lessard P, Carlué M Gomord V, Faye L, Maftah A. The presence of Lewis a epitopes in Arabidopsis thaliana glycoconjugates depends on an active a4fucosyltransferase gene. Glycobiology. 2002:12:299-306.

22. Melo NS, Nimtz M, Conradt HS, Fevereiro PS, Costa J. Identification of the human Lewis a carbohydrate motif in a secretory peroxidase from a plant cell suspension culture (Vaccinium myrtillus L.). FEBS Lett. 1997;415:186-91.

23. Fitchette A-C, Cabanes-Macheteau M, Marvin L, Martin B, Satiat-Jeunemaitre B, Gomord V, Crooks K, Lerouge P, Faye L, Hawes C. Biosynthesis and immunolocalization of Lewis a-containing $\mathrm{N}$-glycans in plant cells. Plant Physiol. 1999;121:333-43.

24. Strasser R, Singh Bondili J, Vavra U, Schoberer J, Svoboda B, Glössl J, Léonard R, Stadlmann J, Altmann F, Steinkellner H, Mach L. A unique B1,3galactosyltransferase is indispensable for the biosynthesis of $\mathrm{N}$-glycans containing Lewis a structures in Arabidopsis thaliana. Plant Cell. 2007;19:2278-92.

25. Oppenheimer DG, Herman PL, Sivakumaran S, Esch J, Marks MD. A myb gene required for leaf trichome differentiation in Arabidopsis is expressed in stipules. Cell. 1991;67:483-893.

26. Wilson IB. Identification of a CDNA encoding a plant Lewis-type alpha 1,4-fucosyltransferase. Glycoconj J. 2001;18:439-47. 
27. Ikram S, Durandet M, Vesa S, Pereira S, Guerche P, Bonhomme S. Functional redundancy and/or ongoing pseudogenization among F-box protein genes expressed in Arabidopsis male gametophyte. Plant Reprod. 2014;27:95-107.

28. Abuqamar S, Luo H, Laluk K, Mickelbart MV, Mengiste T. Crosstalk between biotic and abiotic stress responses in tomato is mediated by the AIM1 transcription factor. Plant J. 2009:58:347-60.

29. Xiao J, Cheng H, Li X, Xiao J, Xu C, Wang S. Rice WRKY13 regulates crosstalk between abiotic and biotic stress signaling pathways by selective binding to different cis-elements. Plant Physiol. 2013;163:1868-82.

30. Traw MB, Bergelson J. Interactive effects of jasmonic acid, salicylic acid, and gibberellin on induction of trichomes in Arabidopsis. Plant Physiol. 2003;133:1367-75.

31. Serna L, Martin C. Trichomes: different regulatory networks lead to convergent structures. Trends Plant Sci. 2006;11:274-80.

32. Dai X, Wang G, Yang DS, Tang Y, Broun P, Marks MD, Sumner LW, Dixon RA, Zhao PX. TrichOME: a comparative omics database for plant trichomes. Plant Physiol. 2010;152:44-54

33. Dubos C, Stracke R, Grotewold E, Weisshaar B, Martin C, Lepiniec L. MYB transcription factors in Arabidopsis. Trends Plant Sci. 2010;15:573-81.

34. Ni SM, Meng $L$, Zhao J, Wang XC, Chen J. Isolation and characterization of the trichome-specific AtTSG1 promoter from Arabidopsis thaliana. Plant Mol Biol Rep. 2008;26:263-76

35. Shangguan $X-X, X u$ B, Yu Z-X, Wang L-J, Chen X-Y. Promoter of a cotton fibre MYB gene functional in trichomes of Arabidopsisand glandular trichomes of tobacco. J Exp Bot. 2008;59:3533-42.

36. Pouteau S, Albertini C. The significance of bolting and floral transitions as indicators of reproductive phase change in Arabidopsis. J Exp Bot. 2009;60:3367-77.

37. Chien JC, Sussex IM. Differential regulation of trichome formation on the adaxial and abaxial leaf surfaces by gibberellins and photoperiod in Arabidopsis thaliana (L.) Heynh. Plant Physiol. 1996;111:1321-8.

38. Telfer A, Bollman KM, Poethig RS. Phase change and the regulation of trichome distribution in Arabidopsis thaliana. Development. 1997;124:645-54.

39. Yu N, Cai WJ, Wang S, Shan CM, Wang LJ, Chen XY. Temporal control of trichome distribution by microRNA156-targeted SPL genes in Arabidopsis thaliana. Plant Cell. 2010;22:2322-35.

40. Alonso JM, Ecker JR. Moving forward in reverse: genetic technologies to enable genome-wide phenomic screens in Arabidopsis. Nat Rev Genet. 2006;7:524-36

41. Boyes DC, Zayed AM, Ascenzi R, McCaskill AJ, Hoffman NE, Davis KR, Görlach J. Growth stage-based phenotypic analysis of Arabidopsis: a model for high throughput functional genomics in plants. Plant Cell. 2001;13:1499-510.

42. D'Agostino IB, Deruère J, Kieber JJ. Characterization of the response of the Arabidopsis response regulator gene family to cytokinin. Plant Physiol. 2000;124:1706-17.

43. Stavang JA, Gallego-Bartolomé J, Gómez MD, Yoshida S, Asami T, Olsen JE, García-Martínez JL, Alabadí D, Blázquez MA. Hormonal regulation of temperature-induced growth in Arabidopsis. Plant J. 2009;60:589-601.

44. Sato H, Mizoi J, Tanaka H, Maruyama K, Qin F, Osakabe Y, Morimoto K, Ohori T, Kusakabe K, Nagata M, Shinozaki K, Yamaguchi-Shinozaki K. Arabidopsis DPB3-1, a DREB2A interactor, specifically enhances heat stress-induced gene expression by forming a heat stress-specific transcriptional complex with NF-Y subunits. Plant Cell. 2014;26:4954-73.

45. Kim SJ, Kim WT. Suppression of Arabidopsis RING E3 ubiquitin ligase AtATL78 increases tolerance to cold stress and decreases tolerance to drought stress. FEBS Lett. 2013;587:2584-890.

46. Blanco F, Salinas P, Cecchini NM, Jordana X, Van Hummelen P, Alvarez ME, Holuigue L. Early genomic responses to salicylic acid in Arabidopsis. Plant Mol Biol. 2009;70:79-102

47. Pieterse CM, van Wees SC, Hoffland E, van Pelt JA, van Loon LC. Systemic resistance in Arabidopsis induced by biocontrol bacteria is independent of salicylic acid accumulation and pathogenesis-related gene expression. Plant Cell. 1996:8:1225-37.

48. Audenaert K, De Meyer GB, Höfte MM. Abscisic acid determines basal susceptibility of tomato to Botrytis cinerea and suppresses salicylic aciddependent signaling mechanisms. Plant Physiol. 2002;128:491-501.

49. Katagiri F, Thilmony R, He SY. The Arabidopsis thaliana-Pseudomonas syringae interaction. Arabidopsis Book. 2002;1:e0039.

50. Goda H, Sasaki E, Akiyama K, Maruyama-Nakashita A, Nakabayashi K, Li W, Ogawa M, Yamauchi Y, Preston J, Aoki K, Kiba T, Takatsuto S, Fujioka S, Asami T, Nakano T, Kato H, Mizuno T, Sakakibara H, Yamaguchi S, Nambara
E, Kamiya Y, Takahashi H, Hirai MY, Sakurai T, Shinozaki K, Saito K, Yoshida S, Shimada Y. The AtGenExpress hormone and chemical treatment data set: experimental design, data evaluation, model data analysis and data access. Plant J. 2008:55:526-42.

51. Kim SH, Gao F, Bhattacharjee S, Adiasor JA, Nam JC, Gassmann W. The Arabidopsis resistance-like gene SNC1 is activated by mutations in SRFR1 and contributes to resistance to the bacterial effector AvrRps4. PLoS Pathog. 2010;6:e1001172.

52. Nishizawa-Yokoi A, Tainaka H, Yoshida E, Tamoi M, Yabuta Y, Shigeoka S. The 265 proteasome function and $\mathrm{Hsp} 90$ activity involved in the regulation of HsfA2 expression in response to oxidative stress. Plant Cell Physiol. 2010;51:486-96.

53. Besseau S, Li J, Palva ET. WRKY54 and WRKY70 co-operate as negative regulators of leaf senescence in Arabidopsis thaliana. J Exp Bot. 2012;63:2667-79.

54. Espunya MC, De Michele R, Gómez-Cadenas A, Martínez MC. SNitrosoglutathione is a component of wound- and salicylic acid-induced systemic responses in Arabidopsis thaliana. J Exp Bot. 2012;63:3219-27.

55. Van Hove J, Stefanowicz K, De Schutter K, Eggermont L, Lannoo N, Al Atalah B, Van Damme EJM. Transcriptional profiling of the lectin ArathEULS3 from Arabidopsis thaliana towards abiotic stresses. J Plant Physiol. 2014;171:1763-73.

56. Karimi M, Inzé D, Depicker A. GATEWAY ${ }^{T M}$ vectors for Agrobacteriummediated plant transformation. Trends Plant Sci. 2002;7:193-5.

57. Clough SJ, Bent AF. Floral dip: a simplified method for Agrobacteriummediated transformation of Arabidopsis thaliana. Plant J. 1998;16:735-43.

58. Jefferson RA. Assaying chimeric genes in plants: the GUS gene fusion system. Plant Mol Biol Rep. 1987;5:387-405.

59. Marks DM, Betancur L, Gilding E, Chen F, Bauer S, Wenger JP, Dixon RA, Haigler $\mathrm{CH}$. A new method for isolating large quantities of Arabidopsis trichomes for transcriptome, cell wall and other types of analyses. Plant J. 2008:56:483-92

\section{Submit your next manuscript to BioMed Central and we will help you at every step:}

- We accept pre-submission inquiries

- Our selector tool helps you to find the most relevant journal

- We provide round the clock customer support

- Convenient online submission

- Thorough peer review

- Inclusion in PubMed and all major indexing services

- Maximum visibility for your research

Submit your manuscript at www.biomedcentral.com/submit
Biomed Central 